\title{
Prevalence and associations of active trachoma among rural preschool children in Wadla district, northern Ethiopia
}

\author{
Mesfin Wudu Kassaw ${ }^{1 *} \mathbb{D}$, Ayele Mamo Abebe ${ }^{2}$, Kirubel Dagnaw Tegegne ${ }^{3}$, Mikiyas Amare Getu and \\ Woldemichael Tadesse Bihonegn ${ }^{4}$
}

\begin{abstract}
Background: Trachoma is a neglected eye disease and an important cause of preventable corneal blindness. In endemic areas, initial infection can occur in early childhood and following a recurrent episodes, it progresses to scarring and visual impairment. Trachoma disappeared from high income countries through enhancements of hygiene and sanitation but the disease is still a challenge in developing countries. In Ethiopia, data indicate that Amhara is the region with the highest prevalence of active trachoma. The aim of this study was to assess the prevalence and associations of active trachoma among rural preschool children in Wadla district, Amhara region, Ethiopia.
\end{abstract}

Methods: In this study, 596 children were screened for signs of active trachoma by using cluster-sampling technique. Following pre-testing of the survey instrument in a different district, questions about socio-demographic status were delivered for heads of households. Integrated eye care workers, previously trained to undertake trachoma screening for one month, performed eye examination. The logistic regression model was used to look for associations of active trachoma.

Results: The prevalence of active trachoma among rural preschool children in Wadla district was 22\%. Low economic status (adjusted odds ratio [AOR]3.8 (95\%Cl 1.3-11.4), being 37-48 months old (4.2;1.5-12.0), living in a house with thatched roof (4.4;1.4-13.6), presence of flies in a home (4.6;2.1-9.9), once-weekly face-washing frequency (8.6;2.5-29.3), having a face that had not been washed for longer than a week (10.6;2.9-37.7), and not using soap (4.5;1.8-11.3) had association to active trachoma.

Conclusion: The prevalence of active trachoma among rural pre-school children in Wadla district was high. This indicates that Trachoma is still a public health problem in the district. This high prevalence calls for further interventions to prevent future trachomatis blindness.

Keywords: Active trachoma, Associations, Water, Sanitation, Hygiene, Wadla district, Ethiopia

\section{Background}

Trachoma is a neglected eye disease and an important cause of preventable corneal blindness [1,2], which is categorized into active and cicatricial types of trachoma

\footnotetext{
* Correspondence: mesfine12a@gmail.com

1 Department of nursing, Woldia University, Woldia, Ethiopia

Full list of author information is available at the end of the article
}

$[2,3]$. In endemic areas, cycles of infection with Chlamydia trachomatis progress to scarring, trachomatis trichiasis, and corneal opacity [4-6]. Trachoma is a disease of poverty and poor hygiene [7] that found primarily in children [4], with the late-stage disease more frequently seen in adult women than adult men, possibly because of women's greater time spent in proximity to

C C The Author(s). 2020 Open Access This article is licensed under a Creative Commons Attribution 4.0 International License, which permits use, sharing, adaptation, distribution and reproduction in any medium or format, as long as you give appropriate credit to the original author(s) and the source, provide a link to the Creative Commons licence, and indicate if changes were made. The images or other third party material in this article are included in the article's Creative Commons licence, unless indicated otherwise in a credit line to the material. If material is not included in the article's Creative Commons licence and your intended use is not permitted by statutory regulation or exceeds the permitted use, you will need to obtain permission directly from the copyright holder. To view a copy of this licence, visit http://creativecommons.org/licenses/by/4.0/. The Creative Commons Public Domain Dedication waiver (http://creativecommons.org/publicdomain/zero/1.0/) applies to the data made available in this article, unless otherwise stated in a credit line to the data. 
children. Pieces of literature indicate that preschool children are the main pool of ocular C. trachomatis infection $[8,9]$.Active trachoma can be an extremely common problem in children, with prevalence estimates of $60-90 \%$ [10]. Ocular C. trachomatis is believed to be transmitted through hand-to-hand contact, sharing of towels, fomites, pillows, and eye-seeking flies [11].

Globally, an estimated 2.5 million people had trachomatis trichiasis, needing surgery $(S)$ to prevent ongoing visual impairment. Another nearly 142 million people lived in districts in which the prevalence of active trachoma met WHO-defined criteria for intervention with antibiotics (A) and interventions to promote facial cleanliness ( $\mathrm{F}$ ) and improve the environment (E), in order to prevent future trichiasis cases. Ethiopia is the most trachoma affected country: more than half of the 142 million people needing the $\mathrm{A}, \mathrm{F}$ and $\mathrm{E}$ components of the "SAFE strategy" in 2019 lived here, Ethiopia [12]. Within Ethiopia, Amhara region has the highest trachoma burden [13], although, the prevalence and associations of active trachoma vary from setting to setting. Hence, studying the differences may help to tailor local control approaches. This is why we undertook investigations in Wadla district, Amhara region after 5 successive years of Zithromax administration in order to reestimate the prevalence of active trachoma and examine its associations.

\section{Methods}

\section{Study design, period and setting}

A community-based cross-sectional study design was used. Fieldwork was undertaken from March 11, 2017 to April 26, 2107. The estimated population of Wadla district was 128,170 with 64,574 males and 63,596 females. There were 28,414 households in this district with an average of 4.5 persons per house. The district had 1 general hospital, 7 health centers, and 20 health posts.

\section{Population}

The sampling frame was children aged 1 to 5 years old in 150 rural villages of Wadla district. The study units were heads from the selected rural households that also had preschool children.

\section{Sample size determination}

We estimated the required sample size using the single population proportion formula. We assumed, based on previous surveys, an observed prevalence of active trachoma (12.1\%) [14], which we wished to estimate with 95\% confidence within $\pm 5 \%$. We used a design effect of 1.5 , and allowed for $10 \%$ non- response rate. Through multiplying the sample size by the design effect, 1.5 and incorporating a $10 \%$ non-response rate, we estimated 273 children that were needed to be framed in selected households.

\section{Sampling technique}

A multistage cluster sampling technique was applied. Wadla district had 20 kebeles (sub-districts) that comprise 247 villages. Twelve of the kebeles were rural, whereas eight of the kebeles were urban. Regarding the villages, 150 of the 247 villages were rural. We used simple random sampling to select 30 of the 150 rural villages. There were 967 households in the selected 30 villages, but only 499 of those households had preschool children. Thus, those 499 households were visited. Heads of households were interviewed for sociodemographic and economic information, plus housing and environmental conditions, and all children aged between 1 and 5 years who had been resident in the district for at least six months were invited to be examined. Eye examiners used the WHO simplified trachoma grading scheme to grade signs of trachoma [15] (Fig. 1).

\section{Definitions \\ Clean face}

A face of child that was free of eye discharges, nose discharges or flies at the time of eye examination.

\section{Preschool}

Children whose age were greater than and equal to 1 year and less than or equal to 5 years old.

\section{Village}

A grouping of homes that contained at least 30 households organized as one peasant association.

\section{Fly in a home}

When there is/are a countable fly in a house during data collection, despite the number of flies.

\section{Active trachoma}

The presence of at least one of the two signs of active trachoma according to the WHO simplified trachoma grading scheme (TF or TI) in at least one eye [16].

\section{Trachomatis inflammation-follicular (TF)}

The presence of five or more follicles each having a diameter of at least $0.5 \mathrm{~mm}$ in the central part of the upper tarsal conjunctiva [16].

\section{Trachomatis inflammation-intense (TI)}

A pronounced inflammatory thickening of the upper tarsal conjunctiva that obscures more than half of the normal deep tarsal blood vessels [16]. 


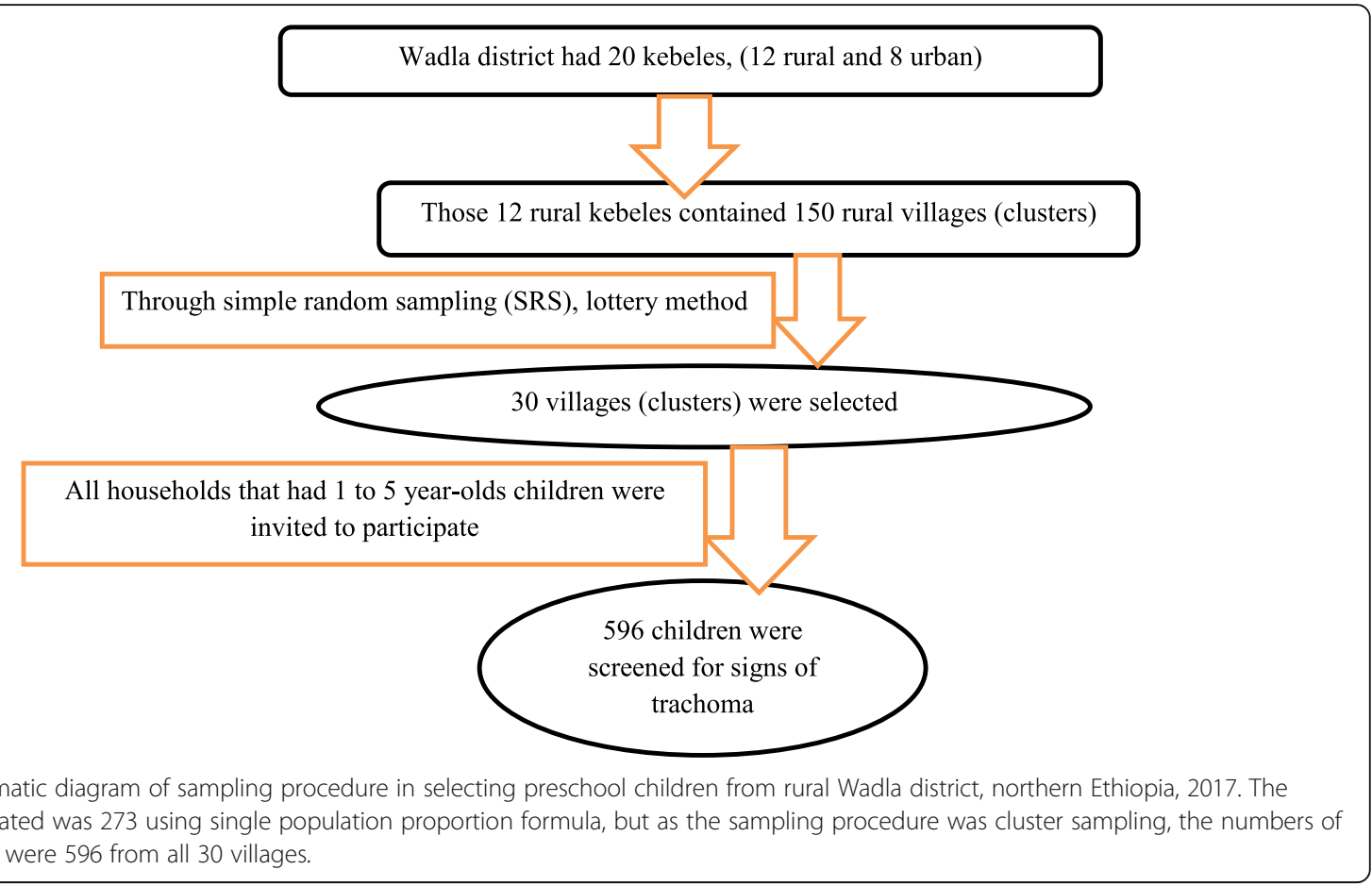

\section{Trachomatis scarring (TS)}

The presence of easily visible scarring in the upper tarsal conjunctiva [16].

\section{Trachomatis trichiasis (TT)}

The presence of at least one eyelash rubs on the eyeball or evidence of removal of in-turned eyelashes in the two weeks before examination [16].

\section{Corneal opacity (CO)}

the presence of easily visible corneal opacity over the pupil [16].

\section{Exclusion and inclusion criteria}

All the children belong to the appropriate age range mentioned above and who had lived in the district for at least 6 months, who were resident in selected villages and available at the time of study were invited to be included. Children who were seriously ill or for whom informed consent was not given by parents or guardians were excluded.

\section{Measurements}

The outcome variable was active trachoma and measured by physical examination. A number of dependent variables were considered that includes sociodemographic, environmental, hygiene and sanitation, and children's demographic data.

\section{Data collection tools and procedures}

In collecting the data, face to face interviews, observation using a checklist and clinical eye examination were used. Experienced health informatics professionals were using structured interview questions that prepared from pieces of literature ( $[17,18]$, and Additional file 1), while they collected the data on a socio-demographic status, environmental, and housing conditions. All the questionnaires of socio-demographic status, housing, and environmental condition, observation checklist, and eye examination tools were pretested and validated before data collection in Kosomender, Meket district, a district bordering Wadla to the north. A household wealth index was developed using composite indicators for rural residents' assets: livestock ownership, size of agricultural land and quantity of crop production.

Two integrated eye care workers performed the eye examination. Those integrated eye care workers are ophthalmic nurses who had been previously trained for a total duration of one month for the purposes of contributing to the 2013-2014national trachoma survey. The Carter Center delivered that previous training using both pictures and live patients as media of instruction. However, for the purpose of this study, the trachoma graders undertook refreshment training for 5 days. This training considers examination of58 live patients and 100 pictures of different trachoma signs. Trainers, whose grades were used as the gold-standard assessment assessed graders. The training was also delivered for interviewers. Interviewers assisted graders by recording clinical 
grades, and data related to each household's sociodemographic status and environmental situation. The trainers emphasized on the objectives, procedures of data collection and mode of communication between graders and interviewers. When undertaking the fieldwork, graders initially observed the eyelashes and cornea of study subjects, looking for TT and CO, then everted the upper lid and inspected the upper tarsal conjunctiva for TF, TI, or TS. Binocular lenses $(\times 2.5)$ and penlight torches were used [4] to magnify the examined eye.

\section{Data analysis and presentation}

The data were checked for completeness, coded and entered into Epi-info version 7, and transferred to SPSS version 23 for analysis. The data were checked for normality using Hosmer-Lemeshow-goodness-of-fit. A univariate analysis model were carried out, and variables that had a $p$-value of $<0.25$ in a binary logistic regression model were included to the multivariate logistic regression analysis. Potential co-linearity was also considered and tested using multi co-linearity model in considering tolerance and variance inflection factor (VIF). Variables with a $p$-value of $<0.05$ in the multivariate logistic regression analysis were considered as statistically significant. A principal component analysis was performed to categorize households' wealth into poorer, poorest, middle, richer, and richest. However, for the presentation of the variables, the wealth index was grouped into three; lowest, middle, and highest. The procedure of eye examination and result reporting presented in Fig. 2. Both active trachoma and cicatricial trachoma were modeled as outcome variables. Thus, children were screened for both Active and cicatricial types of trachoma (Fig. 2).

\section{Data quality assurance}

The questionnaire was prepared in English and translated to Amharic, then re-translated to English (to check for accuracy) by individuals, who are fluent in both English and Amharic. Both graders and one of the researchers, principal investigator had been participated in a community-based trachoma survey and training before starting the present study. The interviewers had also previous experience in a community-based data collection.

\section{Results}

In the study villages, there were 610 preschool children from 499 households. However, only 596 preschool children were examined and gave a response rate of $100 \%$. The remaining 14 children were not involved in the screening phase because of the exclusion criteria and absenteeism after repeated household visit. More than three-fourths $383(77 \%)$ of households had male heads. The range in the number of residents per household was 2 to 10 with a median of five. The range in the number of 1 to 5 years old children per household was 1 to 3 with a median of one. All the 499 families were Amhara in ethnicity and followed Ethiopian orthodox Christianity, and 325 (65\%) fathers, and 380 (76\%) mothers were unable to read and write. Four hundred and sixty-six (93\%) fathers were farmers and 16 (3\%) fathers were government employees (Table 1).

\section{Factors associated with active trachoma}

On binary logistic regression analysis, lowest economic status, being in the age group of 24-36 months old, unable to read and write educational status of fathers, unable to read and write educational status of mothers, living in a house with a thatched grass roof, fly in a house, and a MUAC of children $<13.9 \mathrm{~cm}$ associated with active trachoma (Table 4). However, on the multivariable logistic regression analysis, only lowest economic status (AOR (95\% CI), (3.80 (1.27-11.42)), being 37-48 months old (4.21 (1.47-12.03)), living in a house with a thatched grass roof $(4.40(1.42-13.59))$, or presence of fly in a home (4.6 (2.1-9.9)) were increasing the odds of active trachoma (Table 4).

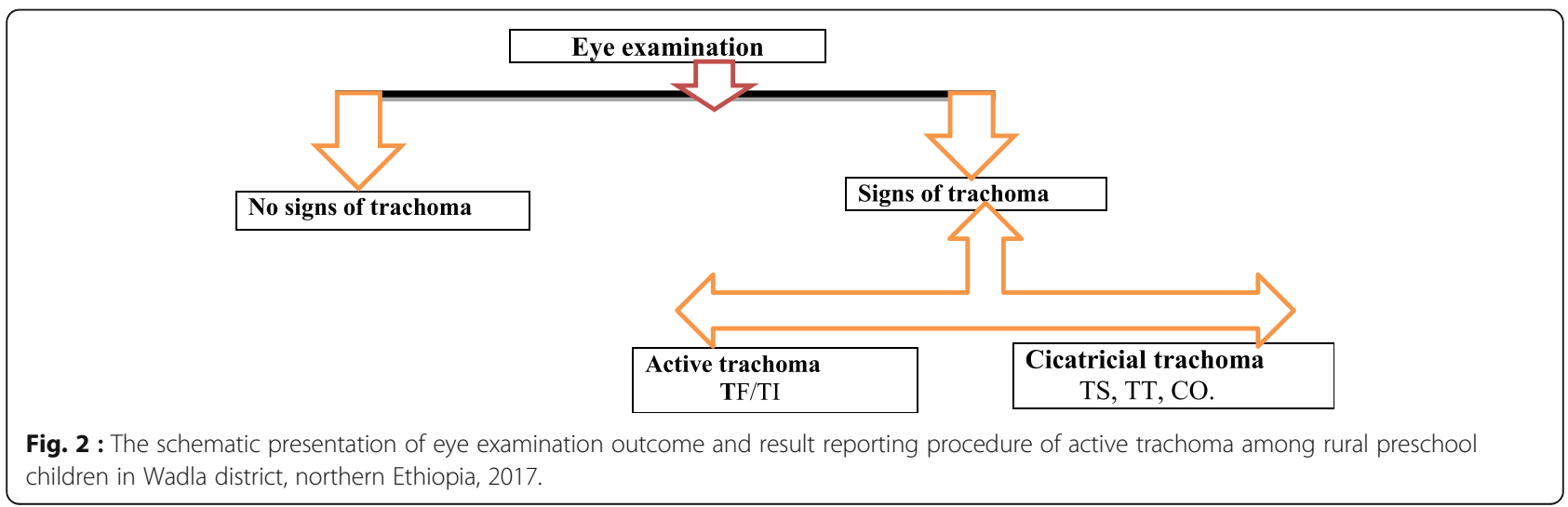


Table 1 Socio-demographic characteristics of heads of households in rural Wadla district, northern Ethiopia, 2017

\begin{tabular}{|c|c|c|}
\hline Variables & Frequency $(n=499)$ & Percent (\%) \\
\hline \multicolumn{3}{|l|}{ Sex of head of a household } \\
\hline Male & 383 & 76.8 \\
\hline Female & 116 & 23.2 \\
\hline \multicolumn{3}{|c|}{ Marital status of head of a household } \\
\hline Married & 492 & 98.6 \\
\hline Divorce & 7 & 1.4 \\
\hline \multicolumn{3}{|l|}{ Wealth index } \\
\hline Poor & 144 & 28.9 \\
\hline Medium & 279 & 55.9 \\
\hline Rich & 76 & 15.2 \\
\hline \multicolumn{3}{|l|}{ Occupation of head of a household } \\
\hline Farmer & 466 & 93.4 \\
\hline Merchant & 17 & 3.4 \\
\hline Government employee & 16 & 3.2 \\
\hline \multicolumn{3}{|c|}{ Educational status of head of a household } \\
\hline Unable to read and write & 325 & 65.1 \\
\hline Able to read and write & 109 & 21.8 \\
\hline Up to grade 8 & 35 & 7 \\
\hline Grade 9 to 12 & 19 & 3.8 \\
\hline Diploma and above & 11 & 2.2 \\
\hline \multicolumn{3}{|l|}{ Educational status of mothers } \\
\hline Unable to read and write & 380 & 76.2 \\
\hline Able to read and write & 55 & 11 \\
\hline Up to grade 8 & 23 & 4.6 \\
\hline Grade 9 to 12 & 35 & 7 \\
\hline Diploma & 6 & 1.2 \\
\hline \multicolumn{3}{|c|}{ Number of rooms in the house (observation) } \\
\hline One & 424 & 85 \\
\hline Two and more & 75 & 15 \\
\hline \multicolumn{3}{|l|}{ Family size } \\
\hline Less than 6 & 286 & 57.3 \\
\hline Greater than and equal to 6 & 213 & 42.7 \\
\hline \multicolumn{3}{|c|}{ Total number of children less than five years in a house } \\
\hline One & 424 & 85 \\
\hline Two & 69 & 13.8 \\
\hline Three & 6 & 1.2 \\
\hline \multicolumn{3}{|c|}{ Number of children less than ten years in a house } \\
\hline One & 132 & 26.5 \\
\hline Two & 240 & 48.1 \\
\hline Three & 102 & 20.4 \\
\hline Four & 25 & 5 \\
\hline \multicolumn{3}{|c|}{ Adult face washing habit (self-report) } \\
\hline At least one times per a day & 417 & 83.6 \\
\hline Less than 7 times per a week & 82 & 16.4 \\
\hline
\end{tabular}

In addition to the socio-demographic characteristics, the environmental characteristics of households are shown in Table 2. 
Table 2 Environmental conditions of the study households in rural Wadla district, northern Ethiopia, 2017

\begin{tabular}{|c|c|c|}
\hline Variables & Frequency $(n=499)$ & Percent (\%) \\
\hline \multicolumn{3}{|l|}{ Presence of fly in or around a house (observation) } \\
\hline Present & 242 & 48.5 \\
\hline Absent & 257 & 51.5 \\
\hline \multicolumn{3}{|l|}{ Source of water (self-report) } \\
\hline River & 30 & 6.0 \\
\hline Unprotected well & 12 & 2.4 \\
\hline Protected well & 56 & 11.2 \\
\hline Pipe & 401 & 80.4 \\
\hline \multicolumn{3}{|l|}{ Amount of water in a litter (self -report) } \\
\hline Less than 20 & 180 & 36.1 \\
\hline $20-40$ & 162 & 32.5 \\
\hline $40-60$ & 92 & 18.4 \\
\hline $60-80$ & 49 & 9.8 \\
\hline Greater than 80 & 16 & 3.2 \\
\hline \multicolumn{3}{|l|}{ Total time taken to reach to water source (self-report) } \\
\hline Less than and equal to $1 / 2 \mathrm{~h}$. & 459 & 92 \\
\hline Greater than $1 / 2 \mathrm{~h}$. & 40 & 8 \\
\hline \multicolumn{3}{|l|}{ Place of cooking (observation) } \\
\hline In the same room of living house & 157 & 31.7 \\
\hline In the same house but in a kitchen & 166 & 33.3 \\
\hline A kitchen constructed against outside wall of the house & 3 & 6 \\
\hline Isolated kitchen & 173 & 34.7 \\
\hline \multicolumn{3}{|l|}{ Presence of window in a kitchen (observation) } \\
\hline Yes & 248 & 49.7 \\
\hline No & 251 & 50.3 \\
\hline \multicolumn{3}{|l|}{ Household waste removal (self-report) } \\
\hline Burn it & 312 & 62.5 \\
\hline Bury it & 90 & 18 \\
\hline Dispose in the farm & 93 & 18.8 \\
\hline Dispose in another place & 4 & .8 \\
\hline \multicolumn{3}{|l|}{ Presence of latrine (observation) } \\
\hline Present & 371 & 74.3 \\
\hline Absent & 128 & 25.7 \\
\hline \multicolumn{3}{|l|}{ Presence of feces at open field in nearby a house (observation) } \\
\hline Present & 243 & 48.7 \\
\hline Absent & 256 & 51.3 \\
\hline \multicolumn{3}{|l|}{ Presence of cattle in a household (observation) } \\
\hline Present & 439 & 87.9 \\
\hline Absent & 60 & 12.1 \\
\hline \multicolumn{3}{|l|}{ Cattle sheltering ( $n=439)$ (observation) } \\
\hline In the same room where family lives & 128 & 29.1 \\
\hline In the same living house but in a separate room & 203 & 46.2 \\
\hline Attached shelter against outside of the house & 6 & 1.6 \\
\hline Isolated shelter far from the house & 102 & 23.1 \\
\hline
\end{tabular}

Among children examined for signs of active trachoma, 301 (51\%) were males, and 295 (49\%) were females. The median age of children was 36 months (Table 3). 
Table 3 Socio-demographic characteristics of the pre-school children in rural Wadla district, northern Ethiopia, 2017

\begin{tabular}{lll}
\hline Variables & Frequency $(n=596)$ & Percent \\
\hline Sex of children & 301 & 50.5 \\
Male & 295 & 49.5 \\
Female & & 34.9 \\
Age of children in months (kebele registration book) & 208 \\
$12-24$ & 102 & 17.10 \\
$25-36$ & 129 \\
$37-48$ & 157 & 21.6 \\
$49-59$ & & 26.3 \\
Current breast-feeding status of children & 239 & 40.1 \\
Yes & 357 & 59.9
\end{tabular}

Face washing frequency of children (self-report)

2 or more times per a day 108

Once daily

2 to 6 times per week

Once weekly

Stays unwashed for longer than a week.

Habit of child bathing for at least one times per a week (self-report)

Yes

No

Use of soap for face washing(self-report)

Yes

No

Use of soap for hand washing(self-report)

Yes

No

Face of children on observation (observation)

Clean face

Ocular discharge

Nasal discharge

Flies on the face of child

Ocular and nasal discharge

Ocular and nasal discharge and flies on the face

Presence of another eye problem(self-report)

Yes

No

Type of eye problem $(n=146)$

Discharge

Itching

Excessive tear

Redness of eye

Took drug during mass drug administration in the last year(self-report)

Yes

No

hundred and eighty $(47 \%)$ children had clean face, $89(15 \%)$ had ocular discharge, $75(13 \%)$ had nasal discharge, $34(6 \%)$ had both ocular and nasal discharge and $55(9 \%)$ children had nasal discharge, ocular discharge, and fly on their face. 
Table 4 Association of active trachoma and risk factors among pre-school children in rural Wadla district, northern Ethiopia, 2017

\begin{tabular}{|c|c|c|c|c|}
\hline \multirow[t]{2}{*}{ Variables } & \multicolumn{2}{|c|}{ Trachoma $(n=596)$} & \multicolumn{2}{|l|}{ OR $(95 \% \mathrm{Cl})$} \\
\hline & Presence (\%) & Absence (\%) & COR & AOR \\
\hline \multicolumn{5}{|c|}{ Type of house roof (observation) } \\
\hline Clean iron & $15(11.5)$ & $82(17.6)$ & 1.00 & 1.00 \\
\hline Thatch iron & $24(18.5)$ & $141(30.3)$ & $0.9(0.5-1.9)$ & $0.9(0.3-2.8)$ \\
\hline Clean grass & $27(20.8)$ & $144(30.9)$ & $1.0(0.5-2.0)$ & $0.7(0.2-2.2)$ \\
\hline Thatch grass & $64(49.2)$ & $99(21.2)$ & $3.5(1.9-6.7) *$ & $4.4(1.4-13.6) *$ \\
\hline \multicolumn{5}{|c|}{ Fly in a house or in nearby (observation) } \\
\hline Yes & $96(73.8)$ & $206(44.2)$ & $3.6(2.3-5.5)$ & $4.6(2.1-9.9) *$ \\
\hline No & $34(26.2)$ & $260(55.8)$ & 1.00 & 1.00 \\
\hline \multicolumn{5}{|c|}{ Face washing frequency (self-report) } \\
\hline Two and more times & $9(6.9)$ & $99(21.2)$ & 1.00 & 1.00 \\
\hline Once daily & $2(1.5)$ & $77(16.5)$ & $0.3(0.1-1.4)$ & $0.2(0.03-1.3)$ \\
\hline 2 to 6 times per a week & $15(11.5)$ & $134(28.8)$ & $1.2(0.5-2.9)$ & $1.366(.365-5.114)$ \\
\hline Once weekly & $63(48.5)$ & $104(22.3)$ & $6.7(3.1-14.1) *$ & $8.7(2.6-29.3) *$ \\
\hline Unwashed for a week & $41(31.5)$ & $52(11.2)$ & $8.7(3.9-19.2) *$ & $10.6(2.9-37.7) *$ \\
\hline \multicolumn{5}{|c|}{ Soap for face washing(self-report) } \\
\hline Used & $26(20)$ & $238(51.1)$ & 1.00 & 1.00 \\
\hline Not used & $104(80)$ & $228(48.9)$ & $4.2(2.6-6.7) *>$ & $4.5(1.8-11.3) *$ \\
\hline \multicolumn{5}{|c|}{ Soap for hand washing(self-report) } \\
\hline Used & $35(26.9)$ & $219(47.0)$ & 1.00 & 1.00 \\
\hline Not used & $95(73.1)$ & $247(53.0)$ & $2.4(1.6-3.7) *$ & $1.6(0.8-3.6)$ \\
\hline \multicolumn{5}{|l|}{ Household latrine (observation) } \\
\hline Present & $7(21.2)$ & $364(78.1)$ & 1.00 & 1.00 \\
\hline Absent & $26(78.8)$ & $102(21.9)$ & $2.0(1.3-3.0) *$ & $5.0(2.0-12.9) *$ \\
\hline \multicolumn{5}{|c|}{ Household waste around the house (observation) } \\
\hline Exist & $80(61.5)$ & $214(45.9)$ & $1.9(1.3-2.8) *$ & $3.4(1.6-7.6) *$ \\
\hline Not exist & $50(38.5)$ & $252(54.1)$ & 1.00 & 1.00 \\
\hline \multicolumn{5}{|l|}{ Mothers educational status } \\
\hline Unable to read and write & $111(85.4)$ & $348(74.7)$ & $2.9(1.3-6.6) *$ & $0.8(0.2-3.2)$ \\
\hline Able to read and write & $12(9.2)$ & $53(11.4)$ & $2.1(0.8-5.7)$ & $0.3(0.1-1.6)$ \\
\hline Attend formal education & $7(5.4)$ & 65 (13.9) & 1.00 & 1.00 \\
\hline \multicolumn{5}{|l|}{ Wealth index } \\
\hline Poor & $73(56.2 \%)$ & $101(21.7)$ & $4.6(2.3-9.1) *$ & $4.2(1.5-12.0)$ \\
\hline Medium & $45(34.6 \%)$ & $288(61.8)$ & $1.003(.506-1.988)$ & $0.5(0.2-1.4)$ \\
\hline Rich & $12(9.2 \%)$ & $77(16.5)$ & 1.000 & 1.00 \\
\hline \multicolumn{5}{|l|}{ MUAC of children } \\
\hline Less than 13.9 & $81(62.3)$ & $230(49.4)$ & $1.7(1.1-2.52) *$ & $1.3(0.6-2.6)$ \\
\hline Greater than 14 & $49(37.7)$ & $236(50.6)$ & 1.00 & 1.00 \\
\hline \multicolumn{5}{|l|}{ Age of children (in months) } \\
\hline $12-24$ & $42(32.3)$ & $166(35.6)$ & $0.8(0.5-1.3)$ & $0.7(0.3-1.8)$ \\
\hline $25-36$ & $14(10.8)$ & $88(18.9)$ & $0.5(0.3-0.9) *$ & $0.7(0.2-2.1)$ \\
\hline $37-48$ & $36(27.7)$ & $93(20)$ & $1.2(0.7-2.1)$ & 2.7(.1.0-7.2) \\
\hline $49-59$ & 38 (29.2) & $119(25.5)$ & 1,00 & 1.00 \\
\hline
\end{tabular}

Fathers education 
Table 4 Association of active trachoma and risk factors among pre-school children in rural Wadla district, northern Ethiopia, 2017 (Continued)

\begin{tabular}{lllll}
\hline Variables & \multicolumn{2}{l}{ Trachoma $(n=596)$} & & OR $(95 \% \mathrm{Cl})$ \\
\cline { 2 - 5 } & Presence $(\%)$ & Absence (\%) & COR & AOR \\
\hline Unable to read and write & $93(71.5)$ & $299(64.2)$ & $2.3(1.1-4.7)^{*}$ & $1.4(0.3-6.2)$ \\
Able to read and write & $28(21.5)$ & $102(21.9)$ & $1.9(0.9-4.5)$ & $2.1(0.5-9.7)$ \\
Formal education & $9(6.9)$ & $65(13.9)$ & 1.00 & 1.00 \\
\hline * & & &
\end{tabular}

\section{Discussion}

The objective of this study was to assess the current prevalence of active trachoma and to identify its associations among children aged 1 to 5 years old in rural communities of Wadla district. The prevalence of active trachoma in this age group was 22\%, [95\%CI, 18-25\%], whereas the prevalence of TF was $21 \%$. Although the usual indicator age group for determining the need or otherwise for the A, F and $\mathrm{E}$ components of the SAFE strategy is the prevalence of TF in 1 to 9 years-old children, the prevalence that we estimate here suggests that three further years of antibiotic mass drug administration is likely to be required, according to WHO recommendation [19]. However, a study from northern Ethiopia reported that azithromycin mass treatment coverage in 2012 was $92.9 \%$ [20]. That reported mass azithromycin coverage was greater than the minimum coverage set by WHO, 80\% [21]. The prevalence agreed with a review that indicated 70 million people in Ethiopia required MDA. This was the largest need of any other country in the world [22]. The prevalence of TI among 1 to 5 years old children here was $3.4 \%$. Severe inflammatory trachoma is a risk factor for later cicatricial disease, particularly when the sign is observed repeatedly over a time [23]. In our subjects, reportedly face washing once weekly and having a face that had remained unwashed for longer than a week were associated with active trachoma. Similar associations had been seen elsewhere $[18,24]$. We also found that the absence of a toilet or presence of human excreta near to a home increased the odds of there being active trachoma. Recent multi-country observational data support the link between inadequate access to sanitation and the likelihood of active trachoma [25]. In general, the associations that we found agreed with the previous published literature that suggests a strong links between trachoma and environmental factors related to water, sanitation, and hygiene. Some of these associations implicate the fly Muscasorbens, which oviposit in human excreta left exposed on the soil, as an important vector [26-28]. In this study, grassed and thatched house roof (AOR (95\% CI), 4.402 (1.425-13.597) were increasing the odds of active trachoma. This association evidenced from central Ethiopia [29].In this study, not using soap was increasing the odds of active trachoma [(AOR (95\%CI), 4.49 (1.79-
11.29)]. This agreed with studies that were conducted in Dessie city and Gonder, Ethiopia [13, 30]. Unfortunately, we did not have any entomological data for this site. Other limitations of our analyses include our reliance on self-report for many of the exposure variables, and the exclusion of children aged 6 to 9 years old. However, this research estimates the prevalence of active trachoma among preschool children from rural area, and its associations, for the attention of policymakers interested in trachoma elimination in Wadala district, Amhara region, Ethiopia.

\section{Conclusions}

The prevalence of active trachoma among rural preschool children in Wadla district was high, suggesting that active trachoma is still a public health problem in Wadla district. Environmental factors were found to be associated with active trachoma. This might suggest an ongoing need for implementation of the F and E components of the SAFE strategy for trachoma elimination in this district to prevent future trachomatis blindness.

\section{Supplementary information}

Supplementary information accompanies this paper at https://doi.org/10. 1186/s12886-020-01585-9.

\section{Additional file 1.}

\section{Abbreviations}

COR: Crude odds ratio; AOR: Adjusted odds ratio; Cl: Confidence interval; AT: Active Trachoma; SAFE: Surgery, Antibiotics, Facial cleanliness,

Environmental improvement; GET2020: Global elimination of Trachoma by 2020; WHO: World Health Organization; TT: Trachomatis trichiasis;

TF: Trachomatis inflammation-follicular; TI: Trachomatis

inflammation —intense; TT: Trachomatis trichiasis; CO: Corneal opacity;

MUAC: Mid upper arm circumference; MDA: Mass drug administration

\section{Acknowledgments}

We would like to acknowledge nursing department, college of health science, Mekelle University, and Wadla district health office for providing funds. We are also grateful to Mr. Semagn Gubala, Mr. Mulugeta Wodaje, Mr. Matiyas Munye, Mr. Melak Menberu, and Mr. Eskeziaw Abebe for their immense contribution in drafting and finalizing the paper. Lastly, we are thankful to the community of the study area, Wadla district and respondents whose participation made the work possible.

Authors' contributions

For this study MWK, AMA, MAG, KDT, and WTB conceived the title and designed the study, conducting a field study, analyzed the data, critically revising the work and writing the final manuscript. All the authors read and 
approved the final version of this manuscript and agreed to be accountable for all aspects of this work

\section{Funding}

This study was sponsored by Mekelle University, College of Health Sciences, and Wadla district health office, northern Ethiopia. Both of the funders did not contribute in collecting, analyzing, writing, and other scientific content of this paper except the full financial expense.

\section{Availability of data and materials}

The data generated in this study will be available to researchers wishing to use the data for non-commercial purposes by asking the principal investigator Mr., Mesfin Wudu through his e-mail, mesfine12a@gmail.com.

\section{Ethics approval and consent to participate}

The study was approved by the Health Research Ethics Review Committee [HRERC0917/2017], College of Health Sciences, Mekelle University. A written permission to implement the study was obtained from Woldia zonal health department and Wadla district health office. A written informed consent was obtained from children's parents or guardians for interview and examination. Confidentiality was maintained by omitting the name and personal identification of respondents within datasets used for analyses.

\section{Consent for publication}

Not applicable.

\section{Competing interests}

The authors have no competing interests.

\section{Author details}

${ }^{1}$ Department of nursing, Woldia University, Woldia, Ethiopia. ${ }^{2}$ Department of nursing, Debre Berhan University, Debre Berhan, Ethiopia. ${ }^{3}$ Department of nursing, Wollo University, Dessie, Ethiopia. ${ }^{4}$ Department of nursing, Samara University, Samara, Ethiopia.

Received: 22 July 2019 Accepted: 28 July 2020

Published online: 26 August 2020

\section{References}

1. Jawetz, Melnick, and Adelberg, Lange Medical Microbiology. The 24th edition. United States of America, McGraw-Hill. 2007, ISBN / ASIN: 0071476660. P 531.

2. Francis $V$, Turner $V$. Achieving community support for trachoma control, a guide for district health work. In: The Edna McConnell Clark Foundation. New York: WHO United States of America, Helen Keller International; 1995.

3. Mabey DC, Solomon AW, Foster A. Trachoma seminar. Lancet. 2003:362 Available from www.thelancet.com.

4. Imtiaz A, Chaudhry, Yonca, Arat, $\mathrm{O}$ and Waleed Al-Rashed. Trachoma and conjunctivitis, conjunctivitis as a complex and multifaceted disorder. (INTE CH open science. 2011. ISBN: 978-953-307-750-5. Available at: http://www. intechopen.com/books/conjunctivitis-a-complex-and-multifaceteddisorder/ trachoma-and-conjunctivitis.

5. Taylor HR. Trachoma as a blinding scourge from the bronze age to the twentieth century. Australia: Centre for Eye Research; 2008.

6. Gambhir M, Basanez MG, Burton MJ, et al. The development of agestructured model for trachoma transmission dynamics, pathogenesis and control. PLoSNegl Trop Dis. 2009;3(6):e462.

7. Habtamu E, Wondie T, Aweke S, et al. Trachoma and relative poverty: a case-control study, PLoSNegl Trop Dis. 2015:9(11):e0004228.

8. Solomon AW, Holland MJ, Burton MJ, et al. Strategies for control of trachoma: observational study with quantitative PCR. Lancet. 2003; 362(9379):198-204.

9. Last A, Burr S, Alexander N, et al. Spatial clustering of high load ocular chlamydia trachomatis infection in trachoma: a cross-sectional populationbased study. Pathog Dis. 2017.

10. Ngondi J, Onsarigo A, Matthews F, et al. The effect of 3 years of SAFE (surgery, antibiotics, facial cleanliness, and environmental change) strategy for trachoma control in southern Sudan : a cross-sectional study. Lancet. 2006;368(9535):589-95.

11. Last A, Versteeg B, ShafiAbdurahman O, et al. Detecting extra-ocular chlamydia trachomatis in a trachoma-endemic community in Ethiopia: identifying potential routes of transmission. PLoSNegl Trop Dis. 2020;14(3): e0008120. 32130213. https://doi.org/10.1371/journal.pntd.0008120.

12. World Health Organization. WHO Alliance for the global elimination of trachoma by 2020: progress report on elimination of trachoma, 2018. WklyEpidemiol Rec. 2019;29(94):317-28.

13. Yemane, Berhane, Alemayehu, Worku, Abebe, Bejiga. A national survey on blindness, low vision and Trachoma in Ethiopia. Federal Ministry of Health of Ethiopia with a support from a consortium of NGOs, Ophthalmological Society of Ethiopia, and the Ethiopian Public Health Association. Addis Ababa, Ethiopia. September 2006.

14. Muluken A, Mulualem E, Melese Y. Prevalence and Risk Factors of Active Trachoma among Children in Gondar Zuria District, North Gondar, Ethiopia. Prevent Med. 2016;1(1:5).

15. Dr A.D. Négrel/Trachoma. Primary Health Care Level Management of Trachoma. WHO Programme for the Prevention of Blindness / Edna McConnell Clark Foundation (U.S.). Geneva: World Health Organization; 1993;4(2):461-66. [WHO/PBL/93.33]. available from: https://www.who.int/ trachoma/resources/who_pbl_93.33/en/.

16. Thylefors B, Dawson CR, Jones BR, West SK, Taylor HR. A simple system for the assessment of trachoma and its complications. Bull World Health Organ. 1987;65(4):477-83. 0003500800.

17. Endale Berta. Prevalence and risk factors of active trachoma among children of rural south Gonder, Ethiopia. Addis Ababa University, Thesis. 2004.

18. Alemayehu M. Assessing the prevalence of active trachoma among young children in relation to the implementation of safe strategy in Ebinat and East Belesaworeda, North West Ethiopia. Ethiopia: Addis Ababa University, Thesis; 2005.

19. Solomon AW, Zondervan M, Kuper H, Buchan JC, Mabey DC, Foster A. Trachoma control: a guide for program managers. Geneva: World Health Organization; 2006.

20. Zelalem T, Teferi GF. Coverage of azithromycin mass treatment for trachoma elimination in Northwestern Ethiopia: a community based crosssectional study. BMC Ophthalmol. 2018;18:193 https://doi.org/10.1186/ s12886-018-0868-1.

21. Melese M, Chidambaram JD, Alemayehu W, Lee DC, Cevallos V, Zhou Z, Donnellan C, Saidel M, Whitcher JP, Gaynor BD. Feasibility of eliminating ocular chlamydia trachomatis with repeated mass antibiotic treatments. JAMA. 2004:292:721-5.

22. WHO. The $\mathrm{WHO}$ alliancefortheglobaleliminationoftrachomabytheyear 2020 Wkly Epidemiol Rec. 2014;89:421-8.

23. West SK, Munoz B, Mkocha H, Hsieh YH, Lynch MC. Progression of active trachoma to scarring in a cohort of Tanzanian children. Ophthalmic Epidemiol. 2001;8(2-3):137-44

24. $\mathrm{WHO}$. The report during the six meetings of the $\mathrm{WHO}$ alliance for the global elimination of blinding trachoma. WHO, Switzerland, Geneva, 2001.

25. Garn JV, Boisson S, Willis R, et al. Sanitation and water supply coverage thresholds associated with active trachoma: modeling cross-sectional data from 13 countries. PLoS Negl Trop Dis. 2018;12(1):e0006110.

26. Emerson PM, Bailey RL, Mahdi OS, Walraven GE, Lindsay SW. Transmission ecology of the fly Muscasorbens, a putative vector of trachoma. Trans R Soc Trop Med Hyg. 2000;94(1):28-32.

27. Emerson PM, Bailey RL, Walraven GE, Lindsay SW. Human and other feces as breeding media of the trachoma vector Muscasorbens. Med Vet Entomol. 2001;15(3):314-20

28. Miller K, Pakpour N, et al. Pesky trachoma suspect finally caught. Br J Ophthalmol. 2004;88(6):750-1.

29. Ngondi J, et al. Risk factors for active trachoma in children and trichiasis in adults: a household survey in Amhara regional state, Ethiopia. Sci Direct Transact Royal Soc Trop Med Hygiene. 2008;102:432-8.

30. Mahande MJ, Mazigo HD, Kweka EJ. Association between water related factors and active trachoma in Hai district, northern Tanzania. Infect Dis Povert. 2012:1:10

\section{Publisher's Note}

Springer Nature remains neutral with regard to jurisdictional claims in published maps and institutional affiliations. 